http://dx.doi.org/10.21611/qirt.2017.028

\title{
MEASUREMENT AND PREDICTION OF Cladosporium cucumerinum AND Collectotrichum orbiculare USING INFRARED THERMOGRAPHY
}

\author{
Ja Hee. Lee ${ }^{\dagger}$, Sang Im. Oh, Ae Kyung. Lee ${ }^{*}$ \\ Department of Environmental Horticulture, College of Bio-resource Science, Dankook University, \\ Cheonan 31116, Korea \\ †Presenting Author: 242love@hanmail.net \\ "Corresponding Author: akleekr@dankook.ac.kr
}

\begin{abstract}
The purpose of this study was to examine the possibility of early diagnosis on plants' abnormal symptoms caused by blight from Cladosporium cucumerium and Colletotrichum orbiculare which are often found in cucumbers before they are observed with the naked eye through nondestructive and contactless analysis using an infrared thermography camera. According to the results, concerning Cladosporium cucumerium, $5^{\text {th }}$ day after pathogenic fungus inoculation, the region inoculated tended to indicate lower temperature than other regions not inoculated. $9^{\text {th }}$ day after the inoculation, the symptoms were observed with the naked eye. About Colletotrichum orbiculare, seven days after the inoculation, temperature in the region inoculated was getting lower, and $13^{\text {th }}$ day after the inoculation, the symptoms were observed with the naked eye. Therefore, infrared thermography camera can be used to conduct early diagnosis before plants' abnormal symptoms caused by blight are seen with the naked eye.
\end{abstract}

KEYWORDS: Cucumber, Early detection, Infrared thermography camera, Non-contracting, Non-destructive

\section{INTRODUCTION}

This study chose cucumbers among many other horticultural plants and applied non-destructive and non-contracting analysis using an infrared thermography camera in order to provide foundational data about whether plants' abnormal symptoms caused by blight from Colletotrichum orbiculare and Cladosporium cucumerium which are most frequently found in cucumbers can be diagnosed early before they are seen with the naked eye.

\section{FORMAT AND LAYOUT}

The pathogens, Cladosporium cucumerium and Colletotrichum orbiculare, used here were gained from Korean Agricultural Culture Collection(KACC)(Fig. 1A, 1B, 1C). To prevent pollution from pathogens, they were tested in different chambers. For pathogenic fungus inoculation, suspension was made by titrating pathogenic fungus in distilled water as $1 \mathrm{mg} \cdot \mathrm{L}^{-1}$ concentration, and it was divided as $10 \mathrm{uL}$ and then inoculated to the surface of cucumber leaves(Fig. 1D). In each experiment, the pathogenic fungus were inoculated ten times repetitively, but the test proceeded excluding cucumber seedlings not indicating the blight until the end. According to the results of the blight finally found, Cladosporium cucumerium was inoculated five times repetitively, and Colletotrichum orbiculare four times. For recording, infrared thermography camera(FLIR T-640, FLIR Systems, Inc., USA) and digital camera(EOS 60D, Canon, Japan) were arranged in $1 \mathrm{~m}$ internals and filmed cucumber leaves once per two days. The infrared thermography camera have $640 \times 480$ pixels, and their thermal sensitivity was $0.035^{\circ} \mathrm{C}$. The images by infrared thermography camera were analyzed with QuickReport 1.2(FLIR Systems, In., USA). Their temperature changes to blight responses were compared, and according to the characteristics of cucumber leaves, 10 points were designated for examining temperature. Cucumber leaves are wide and palmate and have lobed. [2] With the lobed, 
eight points were designated, and the region pathogenic fungus inoculated in the center was set as 9 , and that not inoculated as 10 for examining temperature in each point.
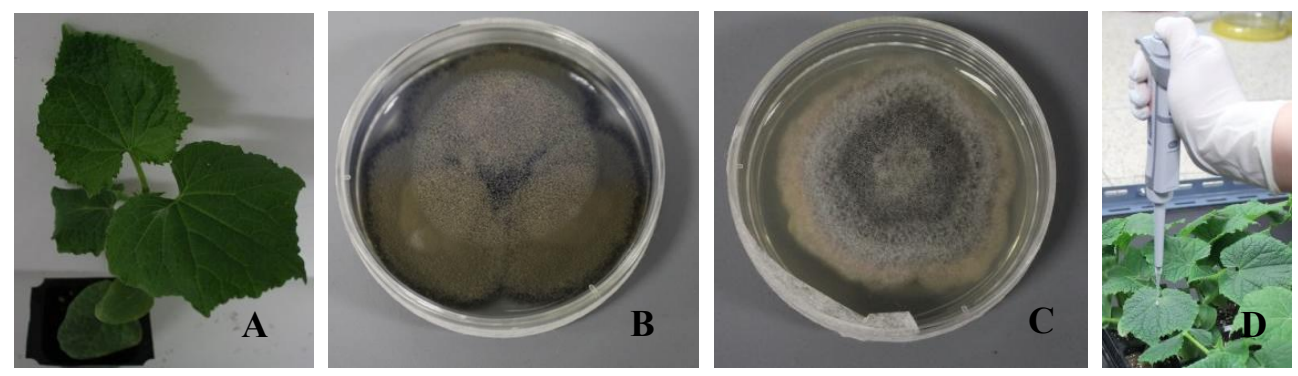

Fig. 1. Materials for experiments

(A: Cucumis sativus, B: Cladosporium cucumerium, C: Colletotrichum orbiculare, D: Inoculating on leaves)

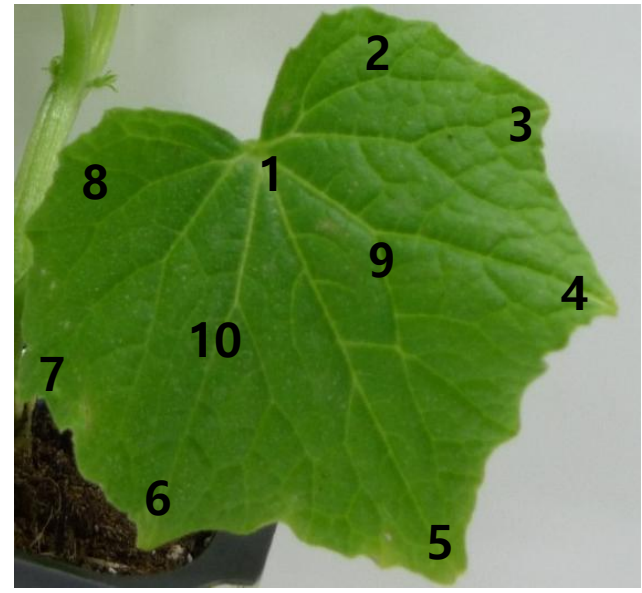

Fig. 2. Point of the lobed in cucumber leaf (1 8: lobed spot of leaf, 9: inoculated, 10: non-inoculated)

\section{CONCLUSIONS}

\section{Thermal imaging analysis on temperature changes caused by Cladosporium cucumerium}

By analyzing infrared thermography images, this study examined the inoculation of Cladosporium cucumerium. According to the results, from 5th day after the inoculation, the regions of leaves inoculated tended to indicate lower temperature than those not. On the $9^{\text {th }}$ day from the inoculation, the symptoms could be seen with the naked eye. The symptoms seen with the naked eye were brown or black blight spots on the leaves infected by Cladosporium cucumerium, and it conforms to the result of Lee, et al. (1997). The thermography images by infrared thermography camera went through analysis with QuickReport to examine the temperature of each region regarding the leaves' lobed or inoculated regions. According to the results, before pathogenic fungus inoculation, cucumber leaves' mean temperature was about $17.77^{\circ} \mathrm{C}$, and right after pathogenic fungus inoculation, the first day of the experiment, the temperature did not indicate significant difference. From the $15^{\text {th }}$ day from the inoculation, the inoculated regions and other regions of the leaves showed significant difference in temperature. Point 9 of pathogenic fungus inoculation indicated about $1.1^{\circ} \mathrm{C}$ lower temperature than other regions. From then until the $25^{\text {th }}$ day, the last day of the experiment, temperature in inoculated regions was maintained rather low. 


\section{Thermal imaging analysis on temperature changes caused by Colletotrichum orbiculare}

$13^{\text {th }}$ day after anthrax bacillus inoculation on cucumbers, the inoculated regions indicated visible symptoms that were brown spots(Fig. 4). The symptoms observed with the naked eye were round yellowish brown blight spots on the leaves which are the same as what has been known as the symptoms of Colletotrichum orbiculare. [3] According to the results of examining temperature changes with infrared thermography images, pathogenic fungus inoculation, cucumber leaves' mean temperature was $16.85^{\circ} \mathrm{C}$, and temperature changes on the regions infected by Colletotrichum orbiculare started $7^{\text {th }}$ day after the pathogenic fungus inoculation. Point 9 , the inoculated region, indicated $15.36^{\circ} \mathrm{C}$ which was about $0.5^{\circ} \mathrm{C}$ different from $15.80^{\circ} \mathrm{C}$, the mean temperature of the other points. After that, inoculated regions showed about $1^{\circ} \mathrm{C}$ difference from non-inoculated regions. Up to the last day of the experiment, the inoculated regions showed low temperature continuously. The $25^{\text {th }}$ day of the experiment was the point when the plants all withered and died, and their temperature was similar to that of the place where the experiment was done.

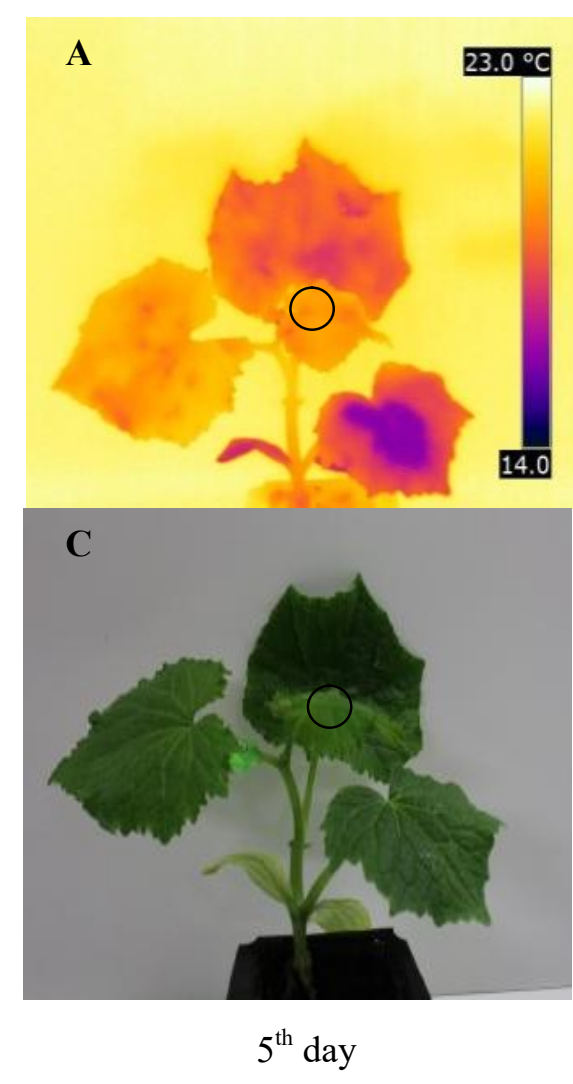

Ambient temperature : $27^{\circ} \mathrm{C}$

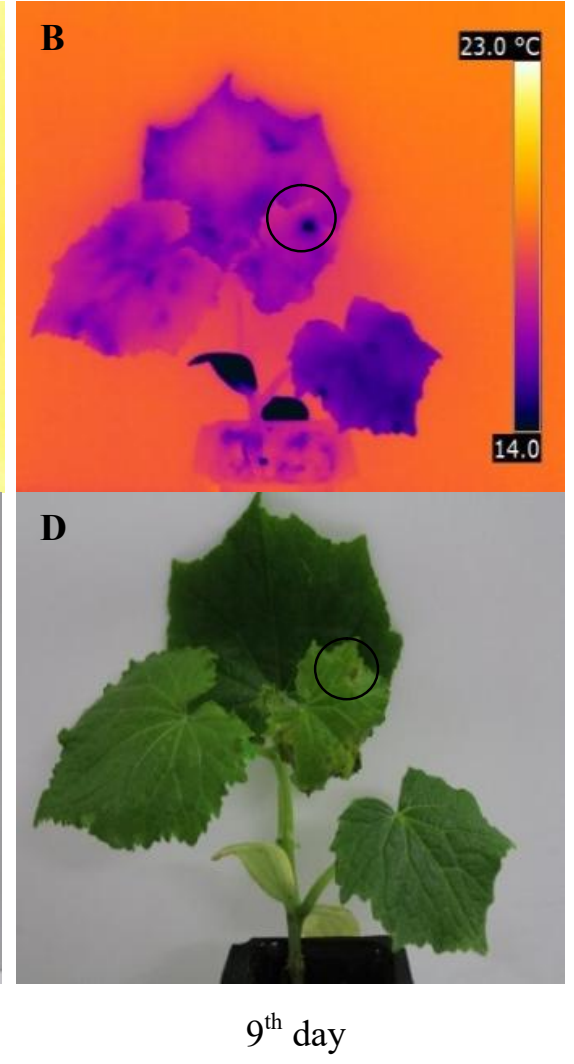

Ambient temperature: $19^{\circ} \mathrm{C}$

Fig. 3. Effect of Cladosporium cucumerium on leaf temperature of cucumber during pathogenesis visualized by infrared thermography camera (A, B: Infrared camera, C, D: Digital camera, A, C : 5 days after inoculated, B, D : 9 days after inoculated). 


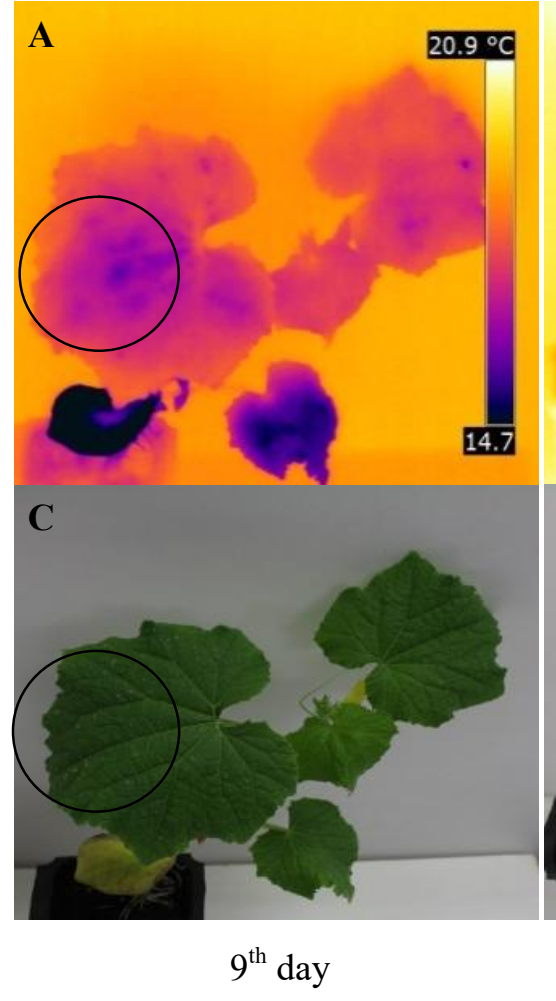

Ambient temperature : $19^{\circ} \mathrm{C}$
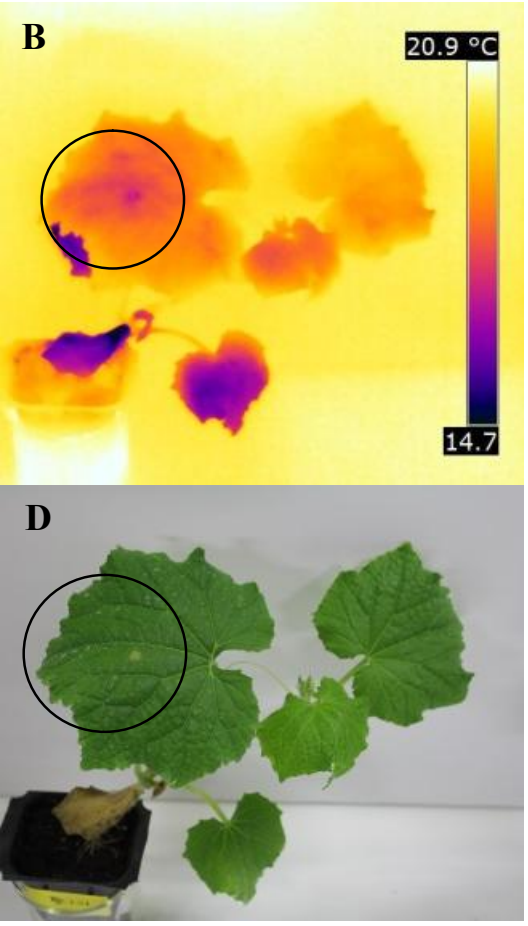

$13^{\text {th }}$ day

Ambient temperature : $22^{\circ} \mathrm{C}$

Fig. 4. Effect of Colletotrichum orbiculare on leaf temperature of cucumber during pathogenesis visualized by infrared thermography camera (A, B: Infrared camera, C, D: Digital camera, A, C : 9 days after inoculated, B, D : 13 days after inoculated).

\section{REFERENCES}

[1] G.D. Ko, Y.C. Kim, I.S. Myung, G.S. Lee, J.S. Lee, J.Y. Lee, B.C. Jang, W.D. Cho, M.J. Han, An illustrated guide to cucumber, Korea agriculture information institute (2010) Korea.

[2] K.Y. Lee, K.H. Youn, H.J. Kang, K.S. Ahn, K.B. Min, B.J. Cha, Cucumber scab caused by Cladosporium cucumerinum in Korea, Korean. J. Plant Pathol 13 (1997) 288-294.

[3] B.A. Auld, M.M. Say, H.I. Ridings, J. Andrews, Field applications of Colletotrichum orbiculare to control Xanthium spinosum, Agric. Ecosyst Enuiron 23 (1990) 315-323. 